obtained (it is best to have a sheet with a short this may indicate a higher presence of chronic résumé of the project in language comprehensible to the layman, which can be signed by the subjects). In addition, the protocol should take full note of the confidentiality required when handling patients' notes and reports, etc.

Account should also be taken of the funding of research projects and, in particular, of monies received by medical staff participating in such projects, and the research project should in no way interfere with the normal services to National Health Service patients, in that it should not remove essential nursing, medical, physiotherapy staff, etc, from their normal commitments.

The nurse member of the committee has proved very useful. As Professor Robinson states, a general practitioner may be invited (essential when the investigator is also a general practitioner), and information to general practitioner colleagues often needs to be considered. Minutes are circulated to all concerned and may help local committees elsewhere in multicentre studies.

N O'Mullane

P F UNSWORTH

Tameside General Hospital,

Lancashire OL6 9RW

\section{Housing conditions and ill health}

SIR,-We read with interest the paper by Dr Claudia J Martin and colleagues (2 May, p 1125). We believe that housing is an important public health issue, not only for children but also for elderly people.

Our group carried out a multidimensional study of a large elderly population (1201 subjects) living in a restricted area in the centre of Brescia, northern Italy; $92 \cdot 2 \%$ of the $70-75$ year old inhabitants in the area were investigated. Data on socioeconomic condition and health were collected. Firstly, we analysed housing conditions (dampness, poor aeration, non-uniform temperature) with poor houses being defined by the presence of one of these negative conditions. Secondly, we analysed health state by asking the elderly subjects how many days they had spent in bed because of illness during the past month and about the presence of common symptoms, such as chest pain, dyspnoea, cough, swollen feet or ankles, tiredness, anorexia, dyspepsia, insomnia, and aches. The data were analysed using the $\chi^{2}$ test.

The data showed a greater prevalence of somatic symptoms in the group of elderly people living in poor houses in comparison with those living in non-poor houses, with significant differences for dyspnoea, tiredness, dyspepsia, insomnia, and aches. Particularly relevant is the difference in the percentage of the elderly affected by dyspnoea as

Percentage (number) of elderly people (aged 70-75) with somatic symptoms and number of days spent in bed owing to illness in past month according to housing conditions

\begin{tabular}{lcc}
\hline & $\begin{array}{c}\text { Poor } \\
\text { houses } \\
(\mathrm{n}=149)\end{array}$ & $\begin{array}{c}\text { Non-poor } \\
\text { houses } \\
(\mathrm{n}=1052)\end{array}$ \\
\hline Chest pain & $13(19)$ & $8(84)$ \\
Dyspnoea & $32(47)$ & $19(194)^{\star}$ \\
Cough & $16(23)$ & $13(132)$ \\
Swollen feet or ankles & $18(26)$ & $12(126)$ \\
Tiredness & $32(47)$ & $20(204)^{\star}$ \\
Anorexia & $29(43)$ & $23(241)$ \\
Dyspepsia & $26(39)$ & $12(120)^{\star}$ \\
Insomnia & $28(42)$ & $17(174)^{\star}$ \\
Aches & $20(30)$ & $11(110)^{\star}$ \\
No of days spent in bed: & & \\
$\quad$ None† & $76(113)$ & $87(910)$ \\
$>1$ & $24(36)$ & $14(142)$
\end{tabular}
${ }^{\star} \mathrm{p}<0.001 ; t \chi^{2}=10.8, p<0.001$ in comparison with $>1$ days
spent in bed. respiratory disease in the group living in poor houses, in agreement with the observations made by $\mathrm{Dr}$ Martin and coworkers. The data also showed a greater prevalence of days spent in bed owing to illness among people living in poor houses (table).

At present it is difficult to state the impact of poor housing on health compared with other socioeconomic variables. We believe, however, that housing conditions have a central role, particularly in the health of the elderly, as this group spends more time in the house than outside it and because of the high sensitivity of the elderly to the quality of the environment, both psychologically and somatically.

RENZO ROZZINI ANGELO BIANCHETTI SIMONE FRANZONI University Centre for the Study of the Elderly, MARCO TRABUCCHI Brescia, Italy

1 Cole TR. Thoughts on old age and the welfare state. Political economy, history, and health policy. I Am Geriatr Soc 1985;33:869-73.

\section{Subcutaneous or intravenous heparin for deep vein thrombosis}

SIR,-The conclusion of Mr M G Walker and coworkers (9 May, p 1189) that subcutaneous calcium heparin is more effective than intravenous calcium heparin in producing thrombolysis in deep vein thrombosis raises several questions and deserves comment.

Firstly, patients with occluding venous thrombi were excluded from the trial. As venous stasis secondary to occluding thrombi is the most frequent symptom leading to the diagnosis of deep vein thrombosis what were the criteria for performing phlebography? The question is important because if primary selection relies on a routine fibrinogen uptake test we might be dealing with a particular population of patients with deep vein thrombosis.

Secondly, Mr Waller and colleagues acknowledge the fact that the mean daily heparin dose was signiticantly less in the intravenous group (24 384 IU) than in the subcutaneous group (29375 IU). This might be because patients in the subcutaneous group had subtherapeutic kaolin cephalin clotting times, as previously shown. ${ }^{1}$ The figure, however, clearly shows that at least during the last five days of heparin treatment the kaolin cephalin clotting time was supratherapeutic in the subcutaneous group, which should have led in principle to a dose reduction. In fact, these findings might reflect that the higher the anticoagulant effect the better the phlebographic result, as previously suggested. ${ }^{2}$

Thirdly, from table III it is obvious that the thrombi that showed significant lysis or reduction in size were located under the knee. The authors' data reported in the results section.

Fourthly, although patients with proved pulmonary embolism were excluded from the trial, the authors conclude that "subcutaneous calcium heparin should be used as the first choice for managing established acute deep vein thrombosis of the legs, whether associated with pulmonary embolism or not."

In view of the considerable practical importance of this issue, an answer to these critical comments seems to be indicated.

HENRI BounameauX

Department of Medicine, BERNARD KRAHENBUHL. University Hospital of Geneva, Switzerland conclusions thus seem to be at variance with the
1 Hull RD, Raskob GE, Hirsh J, et al. Continuous intravenous heparin compared with intermittent subcutaneous heparin in the initial treatment of proximal-vein thrombosis. N Engl 7 Med 1986;315:1109-14.

2 Krahenbuhl B, Simon CA, Bouvier CA, et al. Traitement héparinique. Comparaison entre les voies d'administration intraveineuse et sous-cutenée. Schweiz Med Wochenschr 1979; 109:1322-5

AUTHORS' REPLY,-Firstly, a fibrinogen uptake test was not used in our trial, the decision to implement phlebography in any patient being made on purely clinical grounds. When phlebography yielded positive results and non-occlusive thrombus was greater than $5 \mathrm{~cm}$ in diameter the patient was entered into the trial, provided that other inclusion criteria were met. When the phlebogram yielded positive results and occlusive thrombus was shown the patient was excluded from the trial.

Secondly, while we acknowledged in our paper that there was a significant difference between the mean daily dose of subcutaneous calcium heparin and intravenous sodium heparin, both were nevertheless in the therapeutic range as determined by the kaolin cephalin clotting test. We are unaware of any evidence that the level of anticoagulation in the therapeutic range has any significant influence on the degree of thrombolysis achieved.

Thirdly, table III illustrates very clearly that 18 $(49 \%)$ in the subcutaneous group and $16(67 \%)$ in the intravenous group had thrombi above the knee that underwent lysis or a reduction in size.

Fourthly, our conclusion that subcutaneous calcium heparin should be used as the treatment of choice in managing established acute deep vein thrombosis of the legs, whether associated with pulmonary embolism or not, is based on the results of the trial, which has shown quite clearly that subcutaneous calcium heparin is superior to intravenous sodium heparin.

Though only two patients in the subcutaneous calcium heparin group and three in the intravenous sodium heparin group suffered pulmonary embolism during treatment, there was no difference in clinical outcome, suggesting that intravenous sodium heparin confers no particular advantage in this respect.

\section{G WALKER J W SHaW G J L Thomson J G R CuMMING M LEA THOMAS}

Ninewells Hospital and Medical School,

Dundee DDI 9SY

SIR,-Mr M G Walker and colleagues (9 May, p 1189) have shown an impressive improvement in the rate of thrombolysis in patients with deep venous thrombosis treated with subcutaneous calcium heparin as compared with intravenous sodium heparin. They discuss several factors that may influence this difference. Two, one of which they dismiss briefly, may be of more fundamental importance.

The mobility of a patient with a continuous intravenous infusion is severely restricted in contrast to the patient receiving twice daily subcutaneous injections. Mr Walker and coworkers do not mention the degree of mobility allowed or encouraged in these patients, although they consider mobility to be one of several potential advantages of subcutaneous injection over intravenous infusion. Increased mobility enhances blood flow and may well aid thrombolysis and reduce the extension of existing thrombus.

The second, and possibly more important, difference lies in the treatment itself. Heparin was given for "up to 14 days," and the mean durations or ranges for the two treatment groups are not 